\title{
RAPID GROWTH OF CHARGED PARTICLE MULTIPLICITY IN HIGH ENERGY $\mathrm{e}^{+} \mathrm{e}^{-}$ANNIHILATIONS
}

\author{
TASSO Collaboration
}

R. BRANDELIK, W. BRAUNSCHWEIG, K. GATHER, V. KADANSKY, K. LÜBELSMEYER, P. MÄTTIG, H.-U. MARTYN, G. PEISE, J. RIMKUS, H.G. SANDER, D. SCHMITZ, A. SCHULTZ von DRATZIG, D. TRINES, W. WALLRAFF

I. Physikalisches Institut der RWTH Aachen, Germany ${ }^{9}$

H. BOERNER, H.M. FISCHER, H. HARTMANN, E. HILGER, W. HILLEN, G. KNOP, P. LEU, B. LÖHR ${ }^{1}$, R. WEDEMEYER, N. WERMES, M. WOLLSTADT

Physikalisches Institut der Universität Bonn, Germany ${ }^{9}$

D.G. CASSEL ${ }^{2}$, D. HEYLAND, H. HULTSCHIG, P. JOOS, W. KOCH, P. KOEHLER ${ }^{3}$, U. KÖTZ, H. KOWALSKI, A. LADAGE, D. LÜKE, H.L. LYNCH, G. MIKENBERG ${ }^{4}$, D. NOTZ, J. PYRLIK, R. RIETHMÜLLER, M. SCHLIWA, P. SÖDING, B.H. WIIK, G. WOLF

Deutsches Elektronen-Synchrotron DESY, Hamburg, Germany

R. FOHRMANN, M. HOLDER, G. POELZ, J. RINGEL ${ }^{5}$, O. RÖMER, R. RÜSCH, P. SCHMÜSER II. Institut für Experimentalphysik der Universität Hamburg, Germany ${ }^{9}$

D.M. BINNIE, P.J. DORNAN, N.A. DOWNIE, D.A. GARBUTT, W.G. JONES, S.L. LLOYD, D. PANDOULAS, A. PEVSNER ${ }^{6}$, J. SEDGBEER, S. YARKER, C. YOUNGMAN

Department of Physics, Imperial College London, England ${ }^{10}$

R.J. BARLOW, R.J. CASHMORE, J. ILLINGWORTH, M. OGG, B. ROE ? , G.L. SALMON Department of Nuclear Physics, Oxford University, England ${ }^{10}$

K.W. BELL, W. CHINOWSKY ${ }^{8}$, B. FOSTER, J.C. HART, J. PROUDFOOT, D.R. QUARRIE, D.H. SAXON, P.L. WOODWORTH

Rutherford Laboratory, Chilton, England 10

Y. EISENBERG, U. KARSHON, D. REVEL, E. RONAT, A. SHAPIRA

Weizmann Institute, Rehovot, Israel 11

J. FREEMAN, P. LECOMTE, T. MEYER, SAU LAN WU, G. ZOBERNIG

Department of Physics, University of Wisconsin, Madison, WI, USA 12

Received 3 December 1979

${ }^{1}$ Now at SLAC, Stanford, CA, USA. ${ }^{2}$ On leave from Cornell University, Ithaca, NY, USA. ${ }^{3}$ On leave from FNAL, Batavia, IL, USA. ${ }^{4}$ On leave from Weizmann Institute, Rehovot, Israel. ${ }^{5}$ Now at CERN, Geneva, Switzerland. ${ }^{6}$ Now at Johns Hopkins University, Baltimore, MD, USA. ${ }^{7}$ On leave from the University of Michigan, Ann Arbor, MI, USA. ${ }^{8}$ Now at the University of California, Berkeley, CA, USA. ${ }^{9}$ Supported by the Deutsches Bundesministerium für Forschung und Technologie. 10 Supported by the UK Science Research Council. ${ }^{11}$ Supported by the Minerva Gesellschaft für die Forschung mbH, Munich. $\quad{ }^{12}$ Supported in part by the US Department of Energy contract WY-76-C-02-0881. 
Hadron production by $\mathrm{e}^{+} \mathrm{e}^{-}$annihilation has been studied for c.m. energies $W$ between 13 and $31.6 \mathrm{GeV}$. As a function of $\ln W$ the charged particle multiplicity grows faster at high energy than at lower energies. This is correlated with a rise in the plateau of the rapidity distribution. The cross section $s \mathrm{~d} \sigma / \mathrm{d} x$ is found to scale within $\pm 30 \%$ for $x>0.2$ and $5 \leqslant W$ $\leqslant 31.6 \mathrm{GeV}$.

Hadron production in $\mathrm{e}^{+} \mathrm{e}^{-}$annihilation proceeds predominantly through the formation of two back to back jets. At very high energies, a new process which leads to the production of three jet events, presumably through hard gluon bremsstrahlung, has been observed $[1,2]$. In the present report we study the evolution with energy of the gross features of hadronic final states produced by $\mathrm{e}^{+} \mathrm{e}^{-}$annihilation.

The experiment was carried out at the DESY $\mathrm{e}^{+} \mathrm{e}^{-}$ storage ring PETRA using the TASSO detector. Data have been taken at c.m. energies $W$ of $13,17,22,27.4$, $27.7,30.0,31.6 \mathrm{GeV}$ and in $20 \mathrm{MeV}$ steps between 29.90 and $31.46 \mathrm{GeV}$ [4]. The detector and the analysis procedure have been described in previous papers $[1,3]$. We repeat a few points pertinent to this study. The hadron events were registered in a large solenoid filled with proportional chambers, drift chambers and scintillation counters for charged particle detection. The efficiency of the track reconstruction program was studied with real data and with Monte Carlo events. The inefficiency for track reconstruction was found to be $3 \%$. Approximately $0.5 \%$ of the reconstructed tracks were spurious. The momentum resolution for charged particles was $\sigma_{p} / p \approx 2 \% \cdot p(p$ in $\mathrm{GeV} / c)$ for momenta above $1 \mathrm{GeV} / c$. In the analysis only tracks were accepted that had a momentum transverse to the axis of the solenoid (= beam axis) of greater than 100 $\mathrm{MeV} / \mathrm{c}$ and reached at least the sixth zero-degree layer of the drift chamber. This imposed an angular cut of

Table 1

Number of accepted events and charged particle multiplicities. The first error given for $\left\langle n_{\mathrm{ch}}\right\rangle$ is the statistical, the second the systematic error.

\begin{tabular}{lcr}
\hline$W(\mathrm{GeV})$ & $\begin{array}{l}\text { Number } \\
\text { of events }\end{array}$ & $\left\langle n_{\mathrm{ch}}\right\rangle$ \\
\hline 13 & 72 & $9.0 \pm 0.4 \pm 0.6$ \\
17 & 38 & $10.7 \pm 0.6 \pm 0.7$ \\
22 & 25 & $11.2 \pm 0.7 \pm 0.8$ \\
27.6 & 132 & $12.1 \pm 0.3 \pm 0.8$ \\
30.3 & 559 & $13.4 \pm 0.2 \pm 0.9$ \\
31.2 & 257 & $13.1 \pm 0.3 \pm 0.9$ \\
\hline
\end{tabular}

$|\cos \theta|<0.87$. A pure sample of hadron events from one-photon annihilation was obtained by requiring at least 4 tracks ( 5 tracks for $W \geqslant 27 \mathrm{GeV}$ ) and the sum of the momenta of the charged particles, $\Sigma\left|p_{i}\right|$, to be above some minimum value which was chosen to be $3 \mathrm{GeV} / c$ at $13 \mathrm{GeV}, 4 \mathrm{GeV} / c$ at $17 \mathrm{GeV}, 5 \mathrm{GeV} / c$ at $22 \mathrm{GeV}$ and $8 \mathrm{GeV} / c$ at the higher energies. After these cuts were made the contamination from beamgas scattering, two-photon processes and $\tau \bar{\tau}$ production was estimated to be negligible. The number of accepted events is given in table 1 . The overall probability that a hadronic event from one-photon annihilation is detected and accepted by the above cuts, was determined with a Monte Carlo program for quark-antiquark production including gluon bremsstrahlung $[5,6]$ and was found to be about $80 \%$. The luminosity was computed from the small angle Bhabha scattering cross section, which was computed using the program of Berends et al. [7].

The data presented below are corrected for acceptance, detection efficiency, momentum cut, radiative effects in the initial state, track losses due to decay or

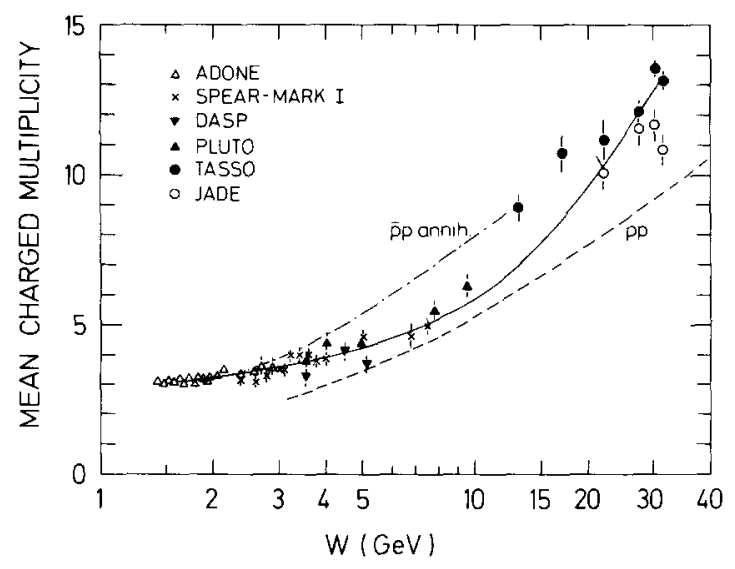

Fig. 1. Average charged particle multiplicity as a function of the c.m. energy $W$ from this experiment ( $\phi$ ) and from other experiments [8-10]. The dashed and dashed-dotted curves show the results for $\mathrm{pp}$ collisions [13] and $\mathrm{p}$ p annihilation [14]. The solid line is a fit to the $\mathrm{e}^{+} \mathrm{e}^{-}$data of the form $\left(n_{\mathrm{ch}}\right\rangle$ $=n_{0}+a \exp \left\{b\left[\ln \left(s / \Lambda^{2}\right)\right]^{1 / 2}\right\}$. 
absorption, and electrons due to photon conversion in 0.13 radiation lengths before the drift chambers. Pions from $K_{s}^{0}$ decays are included. The cross section data shown below have an overall normalization uncertainty of $\sim 15 \%$

Fig. 1 and table 1 show the average multiplicity of charged particles $\left\langle n_{\mathrm{ch}}\right\rangle$ as determined from the observed number of accepted charged tracks after making the corrections described above. The errors shown in fig. 1 for the data from this experiment are statistical only. The systematic errors given in table 1 have been estimated by considering different production mechanisms. Fig. 1 also exhibits measurements of $\left\langle n_{\mathrm{ch}}\right\rangle$ from other experiments [8-12]. The data show that above $\sim 7$ $\mathrm{GeV}$ the multiplicity is rising with $\ln W$ faster than at lower energies. The dashed and dashed-dotted curves in fig. 1 give the energy dependence for $p p$ collisions [13] and $\bar{p} p$ annihilation [14]. The pp multiplicity is about 1 unit below the $\mathrm{e}^{+} \mathrm{e}^{-}$data while the $\bar{p}$ data are slightly above. All three processes have about the same energy dependence.

We have compared different functional forms to describe the data. In the simplest case of scaling $\left\langle n_{\mathrm{ch}}\right\rangle$ $=a+b \ln s$. This does not give a good fit to the data over the full energy range. If low and high energy data are fitted separately to this form one finds

$$
\begin{aligned}
\left\langle n_{\mathrm{ch}}\right\rangle & =(2.67 \pm 0.04)+(0.48 \pm 0.02) \ln s \\
1.4 & \leqslant W<7.0 \mathrm{GeV} \\
\left\langle n_{\mathrm{ch}}\right\rangle & =(-6.1 \pm 0.4)+(2.79 \pm 0.06) \ln s \\
7.0 & \leqslant W \leqslant 31.6 \mathrm{GeV} .
\end{aligned}
$$

The increase in multiplicity due to bottom quark pair production is estimated to be of the order of 0.2 . In QCD the multiplicity is expected [15] to behave as $\left\langle n_{\mathrm{ch}}\right\rangle=n_{0}+a \exp \left\{b\left[\ln \left(s / \Lambda^{2}\right)\right]^{1 / 2}\right\}$. Such a fit to the data $(1.4 \leqslant W \leqslant 31.6)$ yields

$$
\begin{gathered}
\left\langle n_{\mathrm{ch}}\right\rangle=(2.92 \pm 0.04)+(0.0029 \pm 0.0005) \\
\quad X \exp \left\{(2.85 \pm 0.07)\left[\ln \left(s / \Lambda^{2}\right)\right]^{1 / 2}\right\},
\end{gathered}
$$

where $\Lambda$ has been set to $0.5 \mathrm{GeV}$. This fit is shown as the solid line in fig. 1 ; it reproduces the trend of the data.

In order to understand the growth of the multiplicity better, we study the rapidity distribution of charged particle production along the jet axis. The jet axis was determined by the thrust axis ${ }^{\ddagger 1}$; this is obtained by maximizing $\Sigma p_{\| i}$, where $p_{\| i}$ is the longitudinal momentum of a charged particle with respect to this axis, and the summation is extended over all charged particles in a given event. To insure a large acceptance for the particles in the jets, only events were used for which $\left|\cos \theta_{\text {jet }}\right|<0.8$ where $\theta_{\text {jet }}$ is the angle between the jet axis and the beam direction. The effect of this cut on the acceptance was corrected. The rapidity $y$ is defined in terms of the particle energy $E$ and its longitudinal momentum $p_{\|}$by $y=0.5 \ln \left\{\left(E+p_{\|}\right) /\left(E-p_{\|}\right)\right\}$. The maximum of $y$ is $y_{\max } \approx 0.5 \ln \left(\mathrm{s} / \mathrm{m}^{2}\right)$. To compute $E$ the particles were assumed to be pions. Fig. 2a shows the rapidity distribution normalized to the total hadronic cross section, $\sigma_{\text {tot }}$, at 13, 17-22 and 27.4-31.6 $\mathrm{GeV}$. Also plotted are data from SLAC-LBL measured $^{\ddagger 2}$ at low energies (4.8 and $7.4 \mathrm{GeV}$ ) [9]. One observes a plateau near $y=0$ that broadens with increasing c.m, energy. The height of the plateau is not constant but rises with increasing energy (fig. 2b). In the fragmentation region ( $y$ close to $y_{\max }$ ) the particle yield is a steeply descending function of $y$. The width of the fragmentation region is roughly one unit, which is similar to what has been observed in hadron-hadron scattering. In fig. $2 \mathrm{c}$ the same data have been plotted in terms of $y-y_{\max }$ to study the energy behaviour of the fragmentation region. The high-energy data lie systematically below the low-energy data. This is qualitatively what is to be expected from QCD effects. The high-y data should be regarded with some caution because the intrinsic resolution is $\sim 1$ unit due to limited accuracy $\left(\sim 5^{\circ}\right)$ in determining the jet direction.

The energy dependences of the plateau and fragmentation regions show that the faster than logarithmic growth of the average multiplicity at high energies is correlated with a rise of the plateau which is due to low-energy particles. It is interesting to note that the same effect is observed in pp collisions [13].

Next we investigate the energy behaviour of the scaling cross section $s \mathrm{~d} \sigma / \mathrm{d} x$ in terms of the scaled particle momentum $x=p / p_{\text {beam }}$. At particle energies $E$ large enough that particle masses $(m)$ can be neglected,

$\mp 1$ By choosing the thrust direction for the jet axis the corrections for the $y$ distributions relative to the true axis were found to be smaller at large $y$ than if the sphericity axis had been chosen.

$\neq 2$ The SLAC-LBL data used the sphericity axis. 

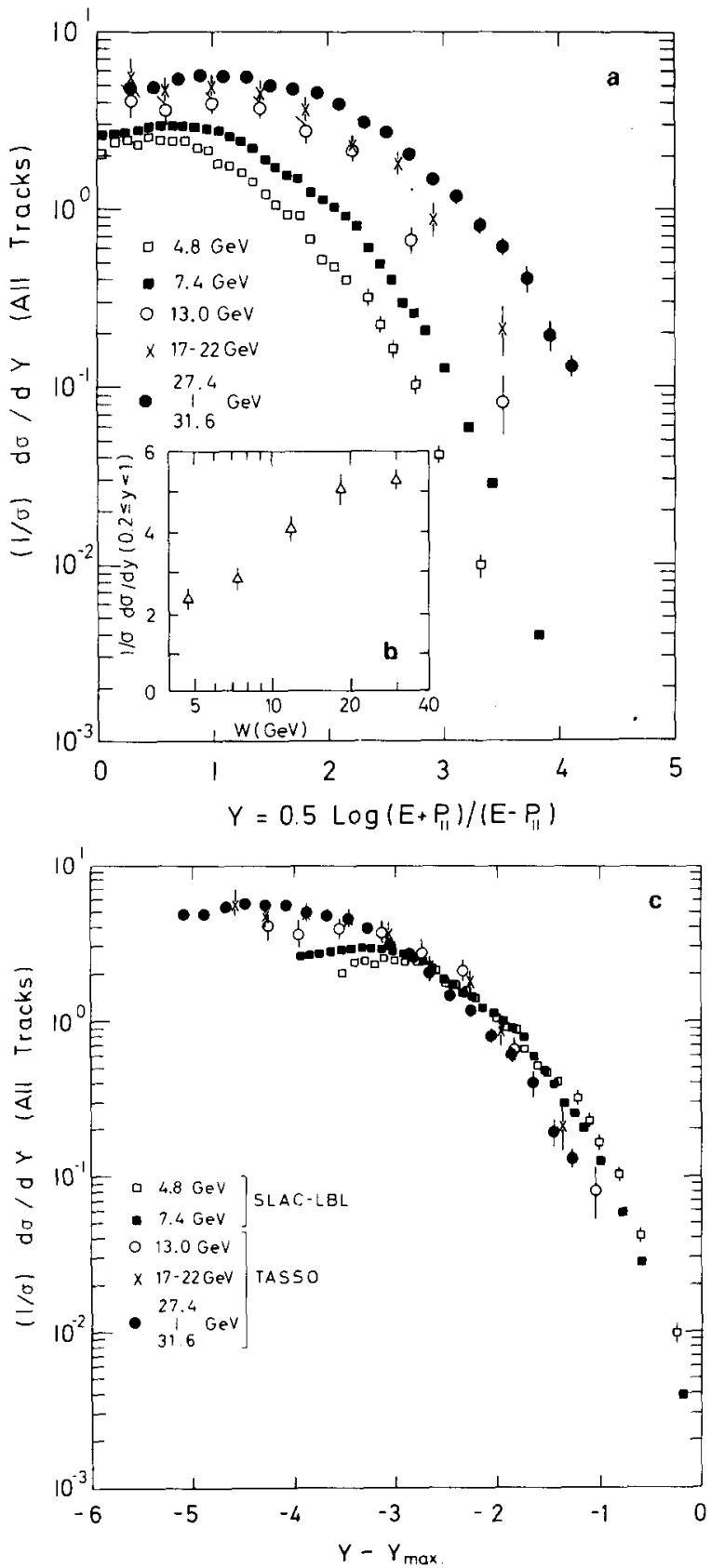

Fig. 2. Rapidity distributions for charged particles (assuming the mass to be the pion mass) measured at 13,17-22 and 27.4 $-31.6 \mathrm{GeV}$ by this experiment, and at 4.8 and $7.4 \mathrm{GeV}$ by SLAC-LBL [9]. For the data of this experiment the thrust axis has been used. In the case of SLAC-LBL the sphericity axis has been used. (a) As a function of $y$; (b) the value of $\left(1 / \sigma_{\text {tot }}\right) \mathrm{d} \sigma / \mathrm{d} y$ averaged over $0.2 \leqslant y \leqslant 1$ as a function of the c.m. energy $W$; (c) the data shown in (a) plotted as a function of $y-y_{\max }$.

the scaling cross section is related to the structure functions $\bar{W}_{1}$ and $\bar{w}_{2}$,

$s \mathrm{~d} \sigma / \mathrm{d} x \approx 4 \pi \alpha^{2} x\left\{m \bar{W}_{1}+\frac{1}{6} x \nu \bar{W}_{2}\right\}$,

where $\nu=\sqrt{s} E / m$ is the photon energy as seen in the particle rest system. The structure functions in general depend on two variables, e.g. $x$ and $s$. If scale invariance holds $\bar{W}_{1}$ and $\nu \bar{W}_{2}$ are functions of $x$ alone and $s \mathrm{~d} \sigma / \mathrm{d} x$ is energy independent.

Fig. 3 displays the data measured in this experiment at $13,17-22$, and $27.4-31.6 \mathrm{GeV}$. For $x>0.2$ they are the same within errors and agree with those measured at low energy by DASP [11] $(5 \mathrm{GeV})$ and SLACLBL [16] $(7.4 \mathrm{GeV})$ to within $\pm 30 \%$. At low $x$ values $(x<0.2)$ the particle yield shows a dramatic rise when the c.m. energy is increased from 5 to $30 \mathrm{GeV}$. This rise is related to the growth of the multiplicity seen above.

Gluon emission will lead to scale breaking effects: the primary momentum is now shared by quark and gluon resulting in a depletion of particles at high- $x$ and an excess of particles at low- $x$ values. The effect becomes more pronounced as the energy becomes

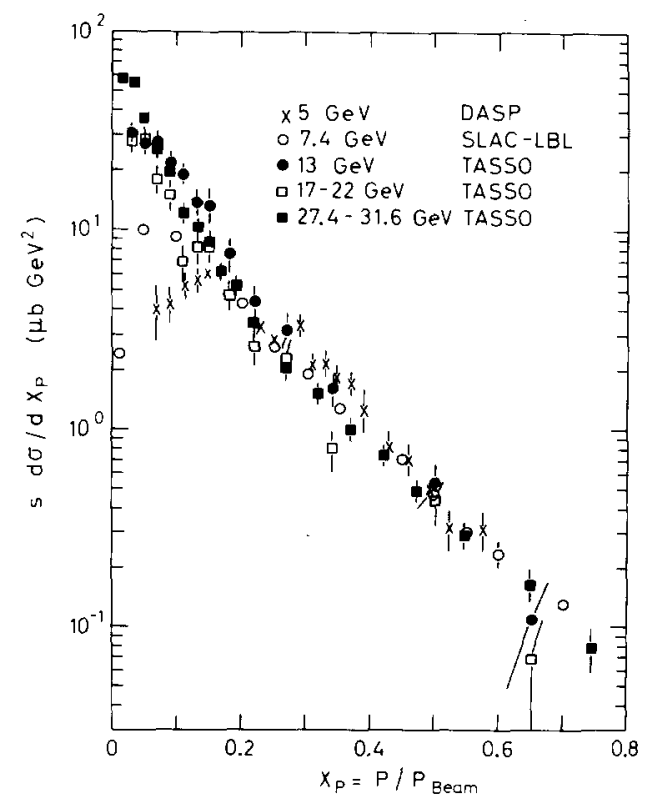

Fig. 3. The scaling cross section $s \mathrm{~d} \sigma / \mathrm{d} x\left(x=p / p_{\text {beam }}\right)$ for inclusive charged particle production measured in this experiment at $13,17-22,27.4-31.6 \mathrm{GeV}$ and at $5 \mathrm{GeV}$ (DASP [11]) and $7.4 \mathrm{GeV}$ (SLAC-LBL [16]). 
larger. More precisely the $30 \mathrm{GeV}$ data for $x=0.2$ are predicted [17] to be higher by $\sim 10 \%$ and for $x=0.7$ lower by $\sim 20 \%$ in comparison with the $5 \mathrm{GeV}$ data. The present data are not precise enough to test this prediction.

In summary the charged particle multiplicity plotted versus $\ln W$ grows faster above $7 \mathrm{GeV}$ than at lower energies. The additional particle yield shows up in the central region of the rapidity distribution. The cross section $s \mathrm{~d} \sigma / \mathrm{d} x$ is consistent with an approximate $( \pm 30 \%)$ scaling behaviour for $x>0.2$ and $5 \leqslant W \leqslant 31.6$ $\mathrm{GeV}$.

An experiment of this type necessarily requires the support of many people, both at DESY and at the collaborating institutions. In particular we would like to thank the PETRA group for their delivery of beam under sometimes difficult circumstances, and the DESY Hallendienst, the support staffs of Rutherford Laboratory, and the Universities for the installation work on tight schedules. The Wisconsin group thanks the Phy. sics Department and especially the High Energy Group and the Graduate School of Research of the University for support. Finally those of us from abroad wish to thank the DESY Directorate for the hospitality extended to us while working at DESY. One of us (P.K.) would like to thank the Alexander von Humboldt-Foundation for support through a Humboldt award.

\section{References}

[1] TASSO Collab., R. Brandelik et al,, Phys. Lett. 86B (1979) 243.

[2] MARK J Collab., Phys. Rev. Lett. 43 (1979) 830;

[3] PLUTO Collab., Phys. Lett. 86B (1979) 418; JADE Collab., Proc. 1979 Intern. Symp. on Lepton and photon interactions.

[3] TASSO Collab,, R. Brandelik et al,, Phys. Lett. 83B (1979) 261.

[4] TASSO Collab., R. Brandelik et al., DESY Report $79 / 75$ (1979).

[5] R.D. Field and R.P. Feynman, Nucl. Phys. B136 (1978) 1.

[6] P. Hoyer, P. Osland, H.G. Sander, T.F. Walsh and P.M. Zerwas, DESY Report 79/21 (1979).

[7] F.A. Berends, K.J.F. Gaemers and R. Gastmans, Nucl. Phys. B68 (1974) 541; B63 (1973) 381.

[8] C. Bacci et al., Phys. Lett. 86B (1979) 234.

[9] SLAC-LBL Collab., G.G. Hanson, 13th Rencontre de Moriond (1978), ed. J. Tran Thanh Van, Vol. III.

[10] PLUTO Collab., Ch. Berger et al., Phys. Lett. 81B (1979) 410 ; V. Blobel, private communication.

[11] DASP Collab., R. Brandelik et al., Nucl. Phys. B148 (1979) 189.

[12] JADE Collab., W. Bartel et al., DESY Report 79/64 (1979).

[13] W. Thomé et al., Nucl. Phys. B129 (1977) 365; see also the review by E. Albini, P. Capiluppi, G. Giacomelli and A.M. Rossi, Nuovo Cimento 32A (1976) 101.

[14] Data given by R. Stenbacka et al., Nuovo Cimento 51A (1979) 63.

[15] J. Ellis, private communication, motivated by W. Furmanski, R. Petronzio and S. Pokorski, Nucl. Phys. B155 (1979) 253.

[16] G.J. Feldman and M.L. Perl, Phys. Rep. 33 (1977) 285.

[17] R. Baier, J. Engels and B. Peterson, Univ. of Bielefeld report BI-TP 79/10 (1979);

W.R. Frazer and J.F. Gunion, Phys. Rev. D20 (1979) 147. 